EUROPEAN ORGANIZATION FOR NUCLEAR RESEARCH

European Laboratory for Particle Physics

Large Hadron Collider Project

LHC Project Report 514

\title{
ELASTIC MODULUS MEASUREMENTS OF THE LHC DIPOLE SUPERCONDUCTING COIL AT 300 K AND AT 77 K
}

\author{
K. Couturier, P. Ferracin, E. Todesco, D. Tommasini and W. Scandale
}

\begin{abstract}
We present measurements of the stress-displacement relation for the superconducting coils used in the Large Hadron Collider main magnets (dipoles and quadrupoles). This mechanical property is relevant to determine the correct amount of azimuthal pre-stress to be imposed on the coil. The hysteresis pattern in the loading and unloading curves is discussed. The stress-displacement curves are used to compute the corresponding elastic moduli and deformations. Measurements are also carried out at liquid nitrogen temperature, using the same framework to interpret experimental data.
\end{abstract}

LHC Division

Presented at the 2001 Cryogenic Engineering Conference and International Cryogenic Materials Conference CEC/ICMC 2001

16-20 July 2001, Madison, Wisconsin, USA

Administrative Secretariat

LHC Division

CERN

CH - 1211 Geneva 23

Switzerland

Geneva, 19 October 2001 


\title{
ELASTIC MODULUS MEASUREMENTS OF THE LHC DIPOLE SUPERCONDUCTING COIL AT $300 \mathrm{~K}$ AND AT $77 \mathrm{~K}$
}

\author{
K. Couturier, P. Ferracin, E. Todesco, D. Tommasini, and W. Scandale \\ CERN (European Laboratory for Nuclear Research) \\ 1211 Geneva 23, Switzerland
}

\begin{abstract}
We present measurements of the stress-displacement relation for the superconducting coils used in the Large Hadron Collider main magnets (dipoles and quadrupoles). This mechanical property is relevant to determine the correct amount of azimuthal pre-stress to be imposed on the coil. The hysteresis pattern in the loading and unloading curves is discussed. The stress-displacement curves are used to compute the corresponding elastic moduli and deformations. Measurements are also carried out at liquid nitrogen temperature, using the same framework to interpret experimental data.
\end{abstract}

\section{INTRODUCTION}

The Large Hadron Collider (LHC), under construction at CERN, the European Organisation for Nuclear Research (Geneva, Switzerland), uses superconducting magnets $[1,2]$, operating at a temperature of $1.9 \mathrm{~K}$, to guide the circulating beam. The main dipole, designed to generate a magnetic field of $8.3 \mathrm{~T}$, has two magnetic apertures contained in a common mechanical structure (see FIGURE 1). The containment structure consists of the austenitic steel collar, the iron yoke and the steel shrinking cylinder, which all confine the coil in fixed geometry and contribute to produce an azimuthal compression, the so-called pre-stress, on the superconducting cables. The pre-stress is imposed to the coil in order to prevent conductor motion, arising from the electromagnetic forces, during the ramp-up of the magnet to the nominal field. To obtain at the end of the magnet assembly the nominal pre-stress, which at ambient temperature is of about $70 \mathrm{MPa}$, it is essential to know the stress-strain relation of the superconducting coil. Moreover, a good knowledge of the rigidity of the coil at ambient and at cryogenic temperature is fundamental to carry out analysis of the thermo-mechanical behaviour of the magnet in the operation condition [47]. 


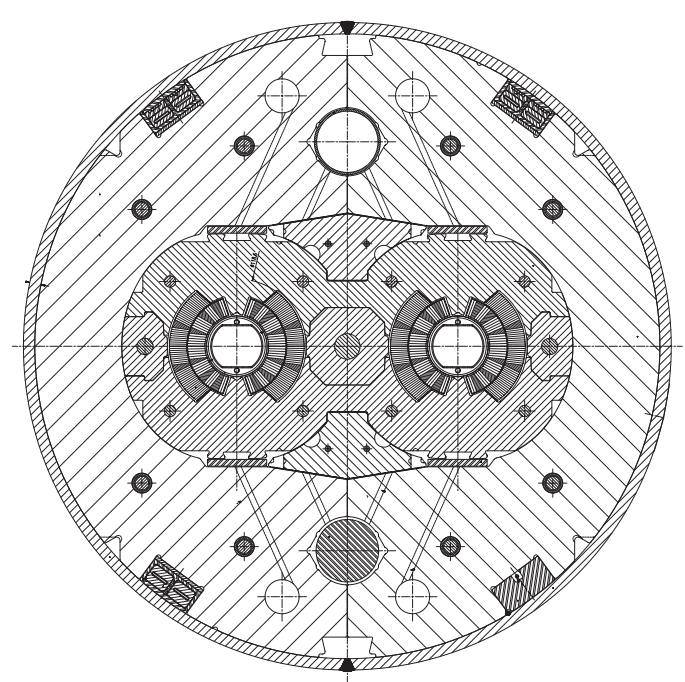

FIGURE 1. Cross-section of the LHC main dipole cold mass.

In this paper we present experimental measurement of the elastic modulus of the coil for the LHC dipole at ambient temperature and at liquid nitrogen temperature. As already observed for other film-insulated superconducting cables [8-17], the coil features a strong stress-dependent rigidity and a stress-displacement curve characterised by a relevant mechanical hysteresis during the loading-unloading compression cycle.

\section{SAMPLE AND EXPERIMENTAL SET-UP}

The coil of the LHC main dipole consists of two layers (inner layer and outer layer) composed by two different keystoned cables (Rutherford type) [18]. The cable width is $15.10 \mathrm{~mm}$ while the mid-thickness at $50 \mathrm{MPa}$ is 1.90 for the inner layer and 1.48 for the outer layer.

Three layers of polyimide tape insulate the conductors, providing channels for helium penetration inside the coil. We measure a stack of 28 outer layer conductors $50 \mathrm{~mm}$ long,

TABLE 1. Measurements of the sample elastic modulus (GPa) at $300 \mathrm{~K}$ and at $77 \mathrm{~K}$ and comparison with the reference values.

\begin{tabular}{lcc}
\hline $300 \mathrm{~K}$ & Measure & Reference \\
\hline Aluminum & $73 \pm 1$ & 70 \\
Copper & $125 \pm 1$ & 120 \\
Invar & $151 \pm 1$ & 150 \\
Steel & $200 \pm 8$ & 195 \\
\hline $77 \mathrm{~K}$ & Measure & Reference \\
\hline Aluminum & $78 \pm 3$ & 77 \\
Copper & $128 \pm 6$ & 138 \\
Invar & $129 \pm 9$ & 150 \\
Steel & $206 \pm 15$ & 209 \\
\hline
\end{tabular}


placed alternately to obtain a parallel side structure for a total height of about $50 \mathrm{~mm}$. The stress-displacement curves are measured by an electro-mechanical apparatus for high precision compression tests (UTS 200.4).

The precision of the displacement measurements is $1 \mu \mathrm{m}$, while the error on the values of the force is of $\pm 10 \mathrm{~N}$. The cross-head speed is $0.05 \mathrm{~mm} / \mathrm{s}$ and it is similar to the compression speed of the coil during the magnet assembly [19]. For the measurement at 77 $\mathrm{K}$ the stack is placed in a cryostat full of liquid nitrogen. The apparatus has been calibrated at ambient temperature and at $77 \mathrm{~K}$ by measuring the elastic modulus of known material (see TABLE 1).

\section{ELASTIC MODULUS AT $300 \mathrm{~K}$}

\section{Experimental procedure and general features}

In FIGURE 1 we plot the stress imposed by the electro-mechanical apparatus at $300 \mathrm{~K}$ versus the total height of the stack. We performed five different loading-unloading cycle with decreasing peak pressure: at the end of each cycle the stress has been reduced to zero, without contact between the cross-head and the stack, in order to obtain a complete relaxation of the stack itself. The stress-displacement curve is characterised by a relevant hysteresis, whose width increases with the peak stress reached during the cycle. Both the loading and the unloading curves are non-linear: the loading curve features a smaller slope, while the slope of the unloading curve varies depending on the peak stress.

\section{Definition of elastic modulus and results}

The elastic modulus at $300 \mathrm{~K}$ can be evaluated as the derivative of the stressdisplacement curve times the unloaded height at $300 \mathrm{~K}\left(l_{w 0}\right)$, that is

$$
E_{w}=\frac{d \sigma_{w}}{d \varepsilon_{w}}=\frac{d \sigma_{w}}{d l_{w}} l_{w 0}
$$

Since, at low pressure, small stress changes give rise to great displacements (see FIGURE 2), the unloaded stack height is ill-defined. Following [20-21], we fix $l_{w 0}$ as the stack height at $0.4 \mathrm{MPa}$ on the loading curve and we obtain (over 15 cycles and assigning a

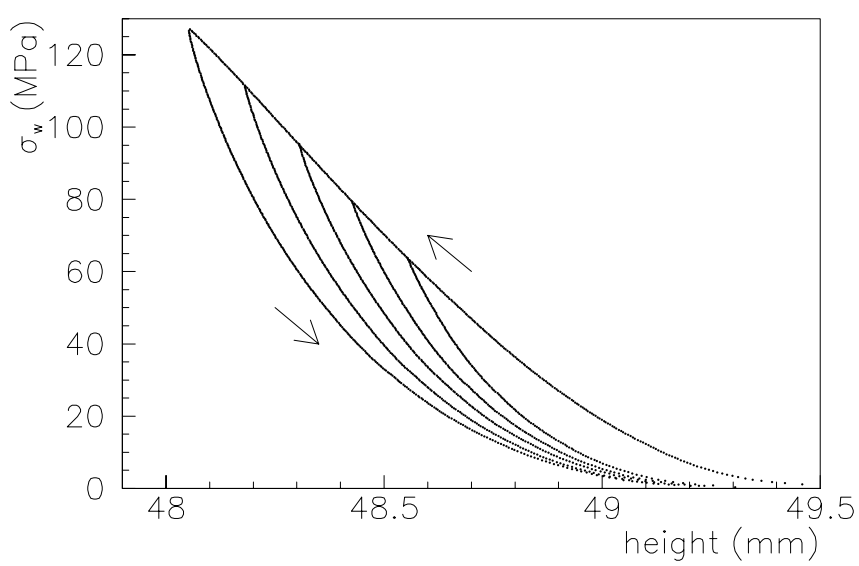

FIGURE 2. Stress $\sigma_{w}(\mathrm{MPa})$ at $300 \mathrm{~K}$ versus total height $(\mathrm{mm})$ for the conductor stack, loading and unloading curves from different peak stresses (experimental data). 


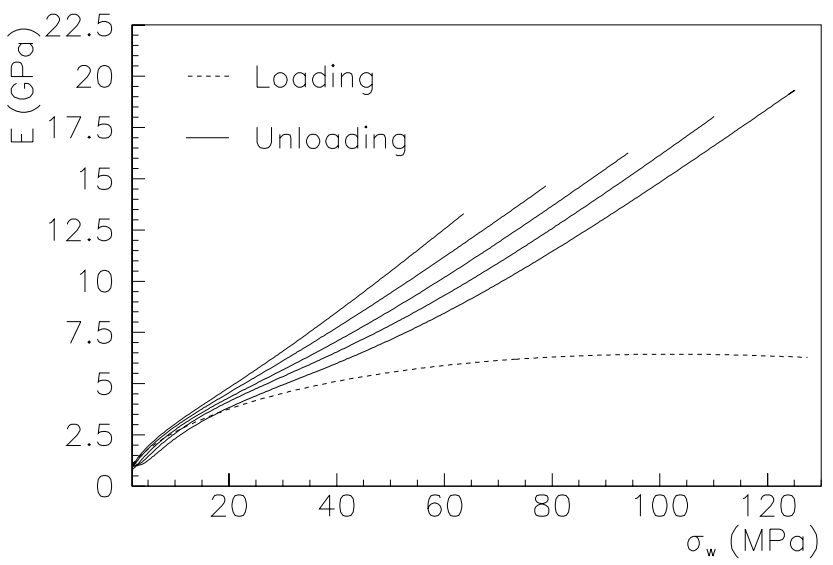

FIGURE 3. Elastic modulus $E(\mathrm{GPa})$ at $300 \mathrm{~K}$ versus stress $\sigma_{w}(\mathrm{MPa})$ evaluated along the loading and the unloading curves.

statistical error of $2 \times$ r.m.s.) a value of $49.51 \pm 0.08$.

In FIGURE 3 we present the evaluation of the elastic modulus: on the loading curve the elastic modulus increases until a compression stress of about $60 \mathrm{MPa}$ and then remains almost constant at a value of about $6.5 \mathrm{GPa}$.

The unloading elastic modulus is much greater and features a strong dependence (almost linear [22]) on the compression stress.

Moreover, an impact of the peak stress on the elastic modulus is observed [23-24]: at the nominal compression stress of $70 \mathrm{MPa}$ it varies from $10.5 \mathrm{GPa}$ to $13.5 \mathrm{GPa}$ depending on the peak stress reached during the cycle, in the range from $65 \mathrm{MPa}$ to $125 \mathrm{MPa}$.

\section{ELASTIC MODULUS AT $77 \mathrm{~K}$}

\section{Experimental set-up, procedure and general features}

Also for the measurements at liquid nitrogen temperature, we performed five different loading-unloading curves with decreasing peak stress.

At $77 \mathrm{~K}$ the hysteresis of the stress-displacement curve (see FIGURE 4) is much smaller than at ambient temperature and the loading branch is characterised by a much smaller slope.

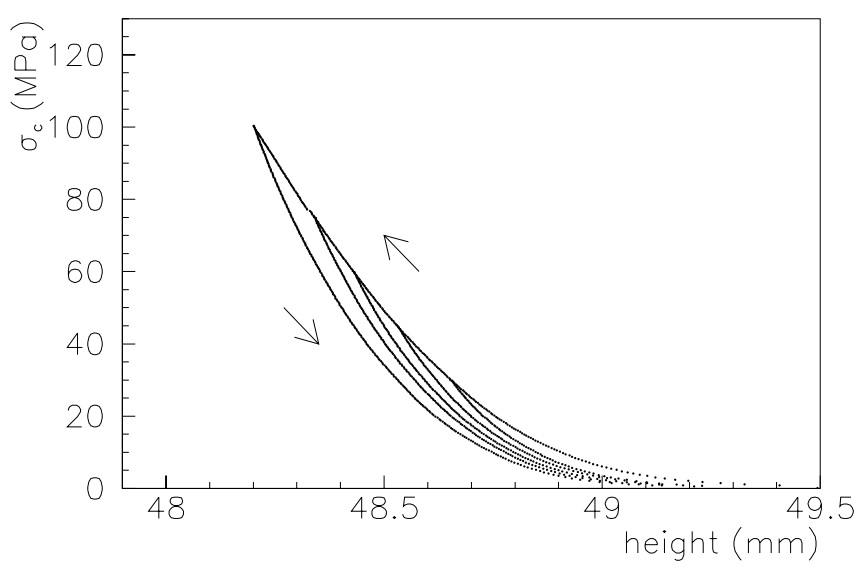

FIGURE 4. Stress $\sigma_{c}(\mathrm{MPa})$ at $77 \mathrm{~K}$ versus total height $(\mathrm{mm})$ for the conductor stack, loading and unloading curves from different peak stresses (experimental data). 


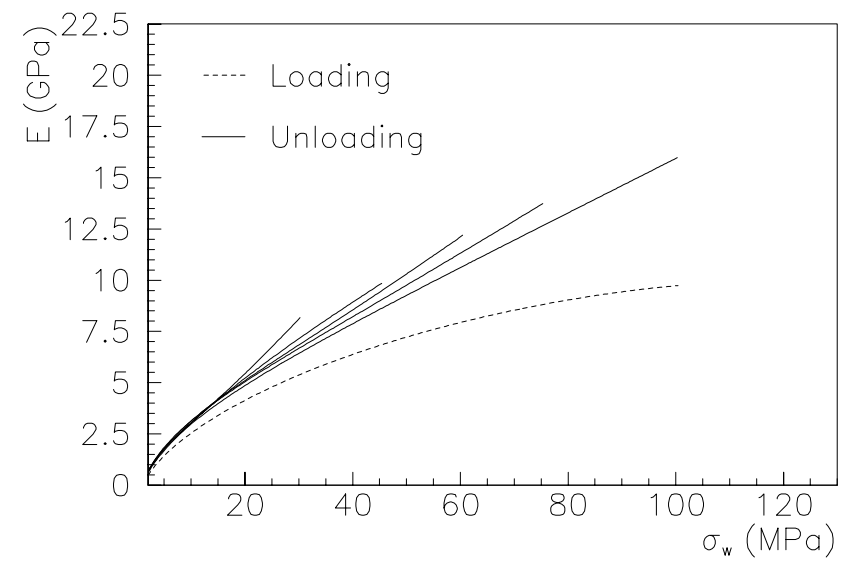

FIGURE 5. Elastic modulus $E(\mathrm{GPa})$ at $77 \mathrm{~K}$ versus stress $\sigma_{c}(\mathrm{MPa})$ evaluated along the loading and the unloading curves.

\section{Definition of elastic modulus and results}

We evaluated the elastic modulus from the stress-displacement curve by

$$
E_{c}=\frac{d \sigma_{c}}{d \varepsilon_{c}}=\frac{d \sigma_{c}}{d l_{c}} l_{c 0}
$$

where $E_{c}, \sigma_{c}$ and $l_{c 0}$ are respectively the elastic modulus, the stress and the unloaded stack height at liquid nitrogen temperature. Since our experimental apparatus doesn't allow a direct measure of the stack height at $77 \mathrm{~K}$, we assume $l_{c 0} \equiv l_{w 0}$.

The difference between the unloaded stack height at ambient and at cryogenic temperature, that is the thermal contraction coefficient, is of the order of $1 \%$ at the total height: therefore, by imposing $l_{c 0} \equiv l_{w 0}$ a negligible error of $1 \%$ is introduced in the evaluation of the elastic modulus.

In FIGURE 5 we plot the results: along the loading curve the elastic modulus is $50 \%$ higher then at ambient temperature. Moreover, the unloading elastic modulus is characterised by similar values with respect to the measurement at 300 , but with a less pronounced dependence on the peak stress.

\section{CONCLUSION}

We presented the measurement of the elastic modulus at $300 \mathrm{~K}$ and at $77 \mathrm{~K}$ performed on a stack of conductors used in the LHC dipole coil, outer layer.

At ambient temperature the stress-displacement curves feature a relevant hysteresis with a non-linear behaviour, both on the loading and on the unloading branch.

The elastic modulus on the loading phase is much smaller than the one on the unloading phase, which presents an almost linear dependence on the compression stress.

At liquid nitrogen temperature, the width of the hysteresis is smaller; the elastic modulus on the loading curve is $50 \%$ higher with respect to the value measured at $300 \mathrm{~K}$, while the elastic modulus on the unloading curve is similar to the one evaluated at ambient temperature. 


\section{ACKNOWLEDGEMENTS}

We would like to thank Stefano Sgobba for useful comments and suggestion on elastic modulus measurements. We wish to acknowledge Arnaud Devred for several discussions on the mechanical properties of superconducting cables.

\section{REFERENCES}

1. Mess, K. -H., Schmuser, P. and Wolff, S., Superconducting Accelerator Magnets, World Scientific, Singapore, 1996.

2. Wilson, M. N., Superconducting Magnets, Oxford, U.K.: Clarendon, 1986.

3. Artoos, K., et al., IEEE Trans. Appl. Superconduct. 10, pp.98-102 (1999).

4. Chapman, M. S., "A Finite Element analysis of an SSC dipole magnet (NC-9 cross-section)", Supercollider 1, Plenum Press, New York, 1989, pp. 659-668.

5. Bajko, M., Fessia, P. and Perini, D., IEEE Trans. Appl. Supercondunct. 10, pp. 81-84 (2000).

6. Dell'Orco, D., "Finite Element analysis of the QC quadrupole for the Superconducting Supercollider", LBL-29600, 1989.

7. Zaslawsky, M., "Nonlinear Finite Element analysis of the SSC superconductivity magnet: including the effects of cooldown, operation, quench, preassembly loads and Lorentz forces", SSC-126, 1987.

8. Thompson, P., et al., IEEE Trans. Nucl. Sci. 28, pp. 3303-3305 (1981).

9. Acerbi, E. and Rossi, L., "Mechanical, thermal and electrical measurements on material and components of the main coils of the Milan superconducting cyclotron", INFN/TC-88/16, 1988.

10. Markley, F. W. and Kerby, J. S., "Investigation of the mechanical properties of superconducting coils", Supercollider 2, Plenum Press, New York, 1990, pp. 753-763.

11. Dixon, I.R., Walsh, R.P., Markiewicz, W.D. and Swenson, C.A., IEEE Trans. Magn. 32, (1996).

12. Saito, T., et al., "Investigation of the mechanical and electrical properties of superconducting coils", Supercollider 5, Plenum Press, New York, 1994, pp. 225-228.

13. Markley, F.W., Kerby, J.S., Sizemore, B., Khoun, C. and King, T., "Investigation of the elastic modulus of SSC coils", Supercollider 3, Plenum Press, New York, 1991, pp. 165-173.

14. Andreev, N. I., Kozub, S. S., Sytnik, V. V., Veshchikov, A. T. and Zlobin, A. V., "Mechanical properties of the coil for the UNK superconducting magnets", IHEP 93-61, Protvino, 1993.

15. Chen, Y., Jayakumar, R. and Yu, K., "Experimental Young modulus calculations", Supercollider 5, Plenum Press, New York, 1994.

16. Zerobin, F., Painer, M. and Eichberger, S., IEEE Trans. Magn. 30, (1994).

17. Sims, R. E., "Tooling and process parameters improvements leading to improved dimensional control of the cured SSC dipole coils", Supercollider 3, Plenum Press, New York, 1991, pp. 57-74.

18. Meuris, C., Baudouy, B., Leroy, D. and Szeless, B., Cryogenics 39, pp. 921-931 (1999).

19. Spigo, G., Private communications, 2001.

20. Meuser, R. B., Caspi, S. and Gilbert, W. S., IEEE Trans. Magn. 17, (1981).

21. Reytier, M., Devred, A., Durante, M., Gourdin, C. and Vedrine, P., "Characterisation of the thermomechanical behaviour of insulated cable stacks representative of accelerator magnet coils", DAPNIASTCM-00-23, 2000.

22. Devred, A., Private communications, 2001.

23. Chichili, D. R., Arkan, T. T., Ozelis, J. P. and Terechkine, I., IEEE Trans. Appl. Superconduct. 10, (2000).

24. Chichili, D. R., Arkan, T. T. and Terechkine, I., "Investigation of cable insulation and mechanical properties of $\mathrm{Nb}_{3} \mathrm{Sn}$ composites", FERMILAB-conf-99/052, 1999. 